\title{
Effect of Polymer Concentration on the Morphology and Mechanical Properties of Asymmetric Polysulfone (PSf) Membrane
}

\author{
N. M. Ismail ${ }^{a^{*}}$, N. R. Jakariah ${ }^{\mathrm{a}}$, N. Bolonga, S. M. Anissuzamanª, N. A. H. M. Nordin ${ }^{\mathrm{b}}$, \\ A. R. Razalic \\ ${ }^{\text {aF }}$ aculty of Engineering, Universiti Malaysia Sabah, 88400 UMS Kota Kinabalu, \\ Sabah, Malaysia \\ bDepartment of Chemical Engineering, Universiti Teknologi PETRONAS (UTP), \\ 32610 Bandar Seri Iskandar, Perak, Malaysia \\ ${ }^{\mathrm{c}}$ Manufacturing Focus Group, Faculty of Mechanical Engineering, Universiti \\ Malaysia Pahang, 26600 Pekan Pahang, Malaysia
}

\begin{abstract}
Polymer concentration had been identified as one of the important parameters tailoring the membrane properties. In this work, the effects of polymer concentration on the morphological and mechanical properties of membrane were investigated at three different polymer concentrations, i.e., 20, 25 and $30 \mathrm{wt} \%$. The viscosity of dope solutions were determined to estimate the optimum polymer concentration. The morphological properties of the fabricated membrane were determined using SEM whereas the mechanical properties of the membrane were investigated using tensile tester. Results show that an increase in the polymer concentration could lead to the improvement of the morphological and mechanical properties of the membrane. The tensile strength of the membrane determined for PSf-20, PSf-25 and PSf-30 are 5.73, 6.59 and 7.03 MPa, respectively whereas the elongation at break measured for the membranes are $46.99 \%, 69.18 \%$ and $36.27 \%$, respectively. As shown in this work, the polymer concentration played a significant role to alter on membrane morphology and mechanical strength.
\end{abstract}

Keywords: Membrane, morphology, mechanical properties, asymmetric flat sheet

\subsection{INTRODUCTION}

Associated gas refers to the natural gas that comes from oil well [1]. This gas can exist either separately from oil in the formation which is known as free gas, or dissolved in the crude oil, also known as dissolved gas. During petroleum crude oil extraction, associated gas will be separated from the crude oil by using a separator as this gas may cause corrosion inside the pipeline. The extracted crude oil will then be further processed before being stored meanwhile the separated associated has to be flared as it is as it contains contaminates majorly carbon dioxide [1]. Basically, the associated gas obtained from oil well is not purely natural gas as it contains other component such as carbon dioxide and hydrogen sulphide. The presence of this contaminates unfortunately lower the energy value of natural gas which lead to a lower selling price [1]. This challenge can be overcome if the natural gas can be successfully purified from the contaminants.

Nowadays, studied had shown that membrane separation is one of the most efficient alternative for gas separation [2]. This is proven as 
membrane technology has comparative advantages over other technologies including compactness and light weight, easier expansion to increase capacity due to modular system, low maintenance, low energy requirements as it does not require phase transition for separation to occurs, low cost, and environmental friendly $[3,4]$.

Asymmetric membrane is preferable morphology for gas separation due to their higher gas productivity [5]. Basically, an asymmetric membrane consists of two layers comprising the dense skin layer that is responsible for the selective separation and a sublayer (porous structure) that functions as a mechanical support for the membrane. The membrane performance depends greatly on the thickness of the dense skin layer of the asymmetric structure. Theoretically, thicker dense layer would result in membrane which is more selective towards $\mathrm{CO}_{2}$, while having lower gas productivity (permeance), and vice versa. Thus, finding the balance between gas selectivity and permeance by controlling the dense layer thickness is very crucial in developing gas separation membrane.

Notably, polymer concentration in a dope solution was identified as the most important parameter for modifying those properties of membrane [6]. This is due to the fact that, increasing polymer concentration in a dope solution would lead to a formation of denser skin layer that has a higher selectivity [6,7]. Ideally, an asymmetric membrane with a hyperthin skin layer will exhibit high permeation property [9].

However, studies had suggested that polymeric membrane which are highly permeable to gases will have a low selectivity and vice versa [10]. This condition remained as the main challenge and motivation for the current study. Thus, it is essential to determine the optimum concentration of the polymer for the improvement of membranes performances for gas separation industry which will lead to optimum selectivity and permeability. Therefore, this study reports on the fabrication of polymeric (PSf) asymmetric membrane by using the phase inversion technique which can be used in the natural gas separation. The objectives of this project are to investigate the effect of polymer concentration on: (a) the viscosity of the dope solution (b) the overall membrane morphology such as micro and macro voids formation with different polymer concentrations (c) mechanical properties of the membrane structure. It was predicted that higher polymer concentration results in higher skin layer thickness, decrease of larger macro-voids within the membrane and improves the membrane mechanical strength.

\subsection{METHODS}

\subsection{Materials}

To fabricate these asymmetric polymeric membrane, Polysulfone (PSfUdel ${ }^{\circledR} \quad$ P-3500), yellowish tranparent granule was purchased from Solvay Plastic. Commercially available n-methyl-2-pyrrolidone

(NMP) supplied by Sigma Aldrich was used as a solvent whereas the non-solvent used for post treatment was methanol supplied by Fisher Scientific.

\subsection{Dope Solution Formulation}

Firstly, polysulfone was dried in an oven with air circulation at $40^{\circ} \mathrm{C}$ for 24 $\mathrm{h}$ to remove any present moist. Secondly, the required corresponding amount of PSf and NMP were weighed using weighing balance. In this work, 
the required polymer concentration to fabricate membrane are $20 \%, 25 \%$ and 30 wt. $\%$. The PSf polymer was poured into the bottle containing NMP solvent. Then, the mixture were stirred at constant speed of $215 \mathrm{rpm}$ using motor stirrer. The polymer was continuously stirred for several hours until a homogeneous solution was formed. Next, the dope solution was immersed in a shaking water bath for approximately $40 \mathrm{~min}$ at room temperature. Finally, the dope solution was left for $24 \mathrm{~h}$ to remove any air bubbles before proceeding with the membrane casting step.

\subsection{Membrane Fabrication}

A small amount of dope solution was poured sufficiently on the top sides of the tapped glass plate. Secondly, the dope solution was spread evenly on the surface of the glass plate which was quickly immersed in a water bath. The membrane formed was transferred and immersed to a different basin containing water bath (tap water) and left overnight with the purpose of removing the solvent left in the membrane. After that, the membrane was immersed in methanol for $4 \mathrm{~h}$ to remove the remaining solvent [11]. Finally, the membrane was dried in a room temperature for 3 days.

\subsection{Membrane Characterization}

The viscosity of dope solutions with five different polymer concentrations of 20, 22.5, 25, 27.5 and $30 \mathrm{wt} \%$ were measured by using (a) Brookfield Programmable DV-II + Viscometer using spindle number 3 whereas, the spindle speed was adjusted according to the dope solution viscosity.

The morphological structures of the fabricated PSf membrane were examined by using scanning electron microscope (SEM) (TM3000, Hitachi).
The cross-section images of the membrane were observed at $15 \mathrm{kV}$ of potential with magnifications of $600 x$, 1500x and 3000x. Firstly, for the samples preparation, the membrane was immersed in liquid nitrogen to break them into a small rectangular shape before being mounted on sample stubs. Then, the samples were coated by using a mixture of gold and platinum in a sputter-coater. To investigate the effect of polymer concentration on the morphological properties of the membrane, the crosssection images of each fabricated membrane samples were analysed by using SEM.

Tensile test on membrane was conducted using a tensile test machine (Shimadzu Trapezium Lite X Version 1.1.2) at room temperature. Three samples with a dimension of $25 \times 50$ $\mathrm{mm}^{2}$ were prepared and tested with a cross head speed of $5 \mathrm{~mm} / \mathrm{min}$. The force at break and difference between the initial length and length at break for each sample were obtained. Finally, the tensile strength and elongation at break values were analysed.

\subsection{RESULTS AND DISCUSSION}

\subsection{Effect of Polymer Concentration on Dope Viscosity}

Result of the viscosity is presented in Figure 1. From this figure, it can be observed that the viscosity of PSf/NMP solution was increased as PSf concentration increased. In addition, the trend of the increasing viscosity was almost doubled from the previous viscosity reading as each of the PSf concentration was increased by $2.5 \%$. This is likely due to polymer chain entanglement that causes the sudden increase in viscosity.

The method of determining the critical PSf concentration is simply by 
extrapolating two straight lines which is drawn based on the slope of the viscosity whereby the viscosity curve becoming more inclined [12]. Then, the critical concentration is determined from the crossed point between the two straight lines. In this work, the critical concentration of PSf polymer is $26 \%$ as shown in Figure 1.

Chung et al. [13] has reported in their work that chain entanglement in a polymeric-solvent mixture plays an important role in tailoring the performance of the membrane. From their work, they suggested that the significant increase in the degree of chain entanglement occurs at a concentration of about $35 \mathrm{wt} \%$ (defined as the critical concentration) for PES/NMP.

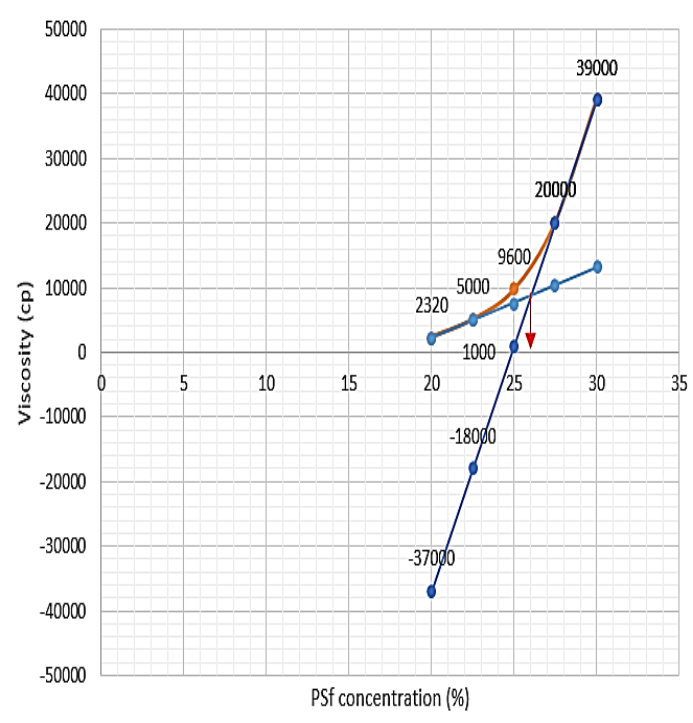

Figure 1 Dope solution viscosity for various concentrations

\subsection{Effect of Polymer Concentration on Morphology}

The effect of polymer concentration on the morphological properties of asymmetric membrane can be observed from the thickness of skin layer formed and the development of macro-voids structures in the porous substructure. Hypothetically, the increase of the polymer concentration in a dope solution will lead to a denser formation of skin layer and lesser and smaller formation of macro-voids structure [14].

In this study, all the fabricated asymmetric PSf membrane presented two distinct layers in their crosssection; the dense skin layer and the support structure as presented in Figure 2 a(i), b(i) and c(i). Moreover, it can be observed that all of the membranes contain macro-voids structure that varies according to the polymer concentration.

The formation of the thin skin layer is due to the instantaneous demixing of solvent and non-solvent during the phase inversion [15]. Furthermore, the development of the large finger-like macro-voids in the porous sublayer is mainly due to the non-solvent diffusion rate into polymer-poor phase is faster than the rate of solvent diffusion outward [16]. Thus, it indicates that the formation of larger finger-like macrovoids is due to the lower polymer concentration. For PSf-25 membrane, (Figure $2 \mathrm{~b}(\mathrm{i})$ and $\mathrm{b}$ (ii)), a thinskinned layer was also observed similar to PSF-20. However, a distinct difference of skin layer thickness between the PSf-20 and PSf-25 membrane cannot be differentiated clearly due to the limitation in the equipment. In addition, finger-like macrovoids observed in the porous sublayer has decreased in length as most of the macro-voids observed are shorter compared to the PSf-20 membrane. However, few large fingerlike macro- voids are still present. This suggests that, an increase in polymer concentration in the dope solution lead to a reduction in the macro-voids structure.

The PSf-30 membrane presented the densest skin layer compared to the PSf-20 and PSf-25 fabricated 
membrane which as shown in the Figure 2 c(i) and c(ii). Additionally, the macro-voids formed in the porous sublayer also decrease in number as

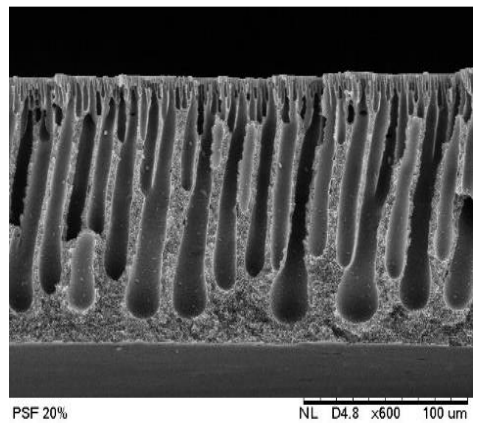

a (i)

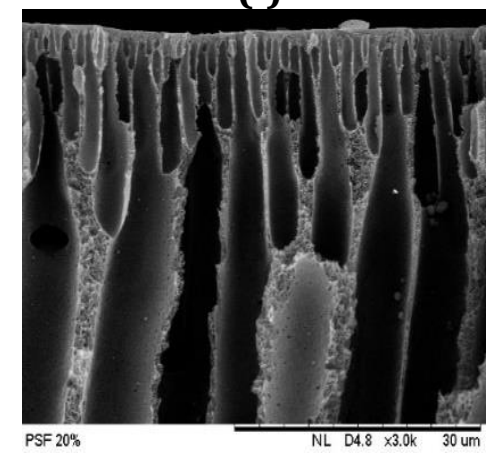

a (ii)

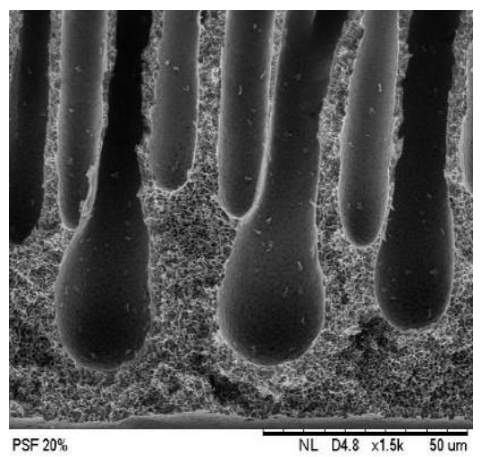

a (iii)

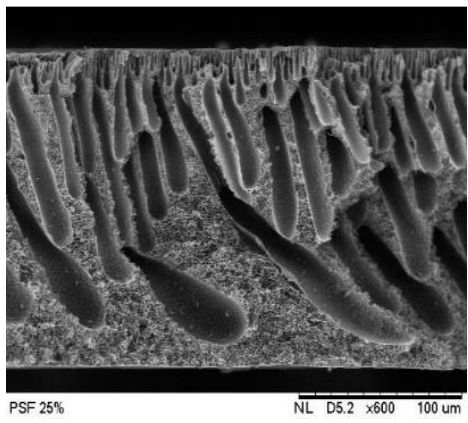

b (i)

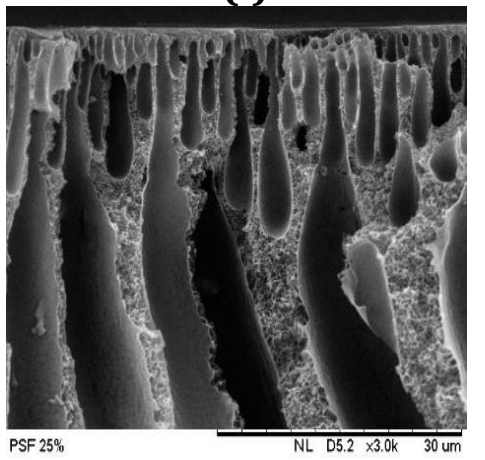

b (ii)

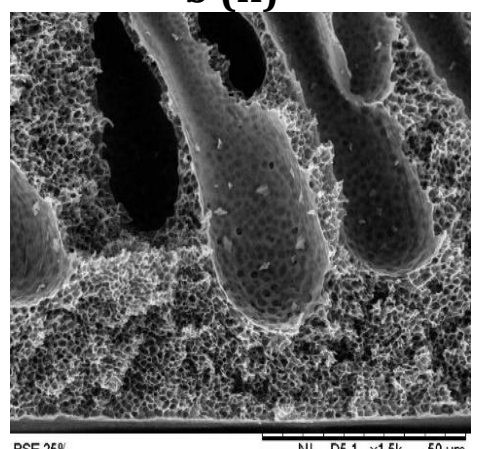

b (iii)

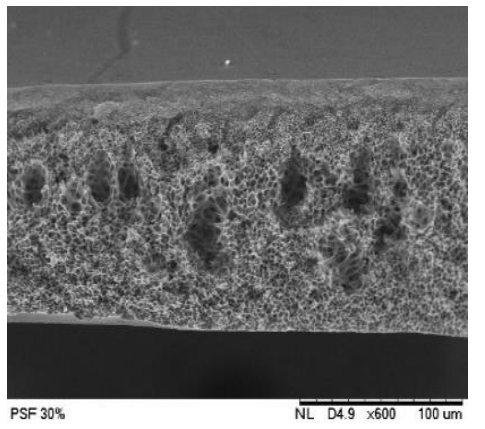

c (i)

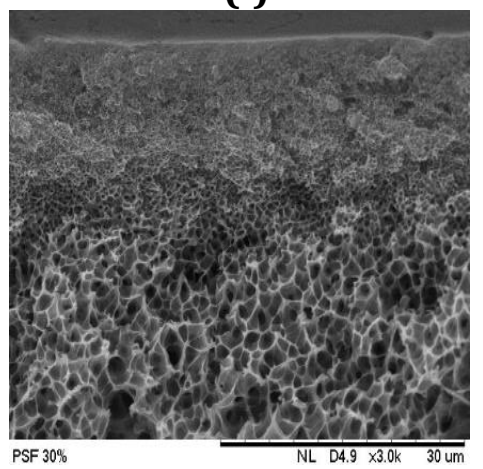

c (ii)

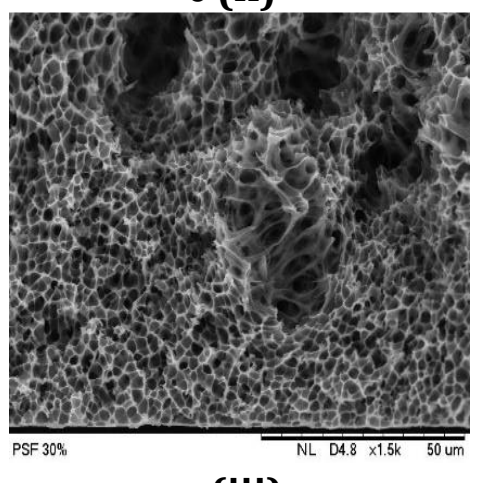

c (iii)

Figure 2 The cross-section of Asymmetric PSf membrane at different polymer concentration; (a) $20 \mathrm{wt} \% \mathrm{PSf}$, (b) $25 \mathrm{wt} \% \mathrm{PSf}$ and (c) $30 \mathrm{wt} \%$ PSf for (i) 500x magnification, (ii) 3,000x magnification and (iii) bottom layer with 1,500x magnification

the concentration of PSf polymer in the dope solution is increased whereas the geometry in which the macro-voids are forming is a teardrop structure which is different compared to the two-previous membrane observed. Further, it can also be noticed that the cross-section of the membrane sublayer porous structure is forming a sponge-like structure as shown in Figure 2 c(iii).
The changes in the morphological structure of the PSf-30 are mainly due to the viscosity of the dope solution that coincides with the higher polymer concentration. This is because, higher viscosity of the dope solution would prevent the diffusion exchange between solvent (NMP) and nonsolvent (water) at the membrane sublayer which lead to the fast phase 
separation at the skin layer and slows precipitation rate at the membrane sublayer occurred [6]. As a result, a denser skin layer is formed as shown in the Figure 2 c(i) and c(ii).

Apart from that, the formation of spongy-like structure of the PSf-30 membrane is also mainly due to the higher viscosity of the dope solution. Considering the kinetics during the diffusion exchange, higher viscosity of dope solution causes demixing to slows down and as a consequence, the membrane morphology changes to a more spongy structure [8]. Overall, the effect of polymer concentration on the morphological properties of the membrane can be observed on the changes occurred in the thickness of the skin layer a well as bottom layer and on the formation of the macrovoids structure. As the polymer concentration increased, the thickness of skin and bottom layer also increased whereas the macro-voids structure changes from a large finger-like structure to a teardrop structure [8].

\subsection{Effect of Polymer Concentration on Mechanical Properties}

The mechanical properties of the membrane different polymer concentration are measured based on the tensile strength and elongation at break to study the effect of polymer concentration on the strength of the membrane as shown Figure 3 and 4. Figure 3 shows the tensile strength result obtained from the tensile test analysis conducted on PSf-20, PSf-25 and PSf-30 membrane. The result shows that an increased in polymer concentration leads to the increased in tensile strength of the membrane. As shown in Figure 3, the tensile strength of the membrane increased about 15\% as the polymer concentration is increased from 20 to $30 \mathrm{wt} \%$ and increased about $23 \%$ when the polymer concentration is increased from 20 to $30 \%$.

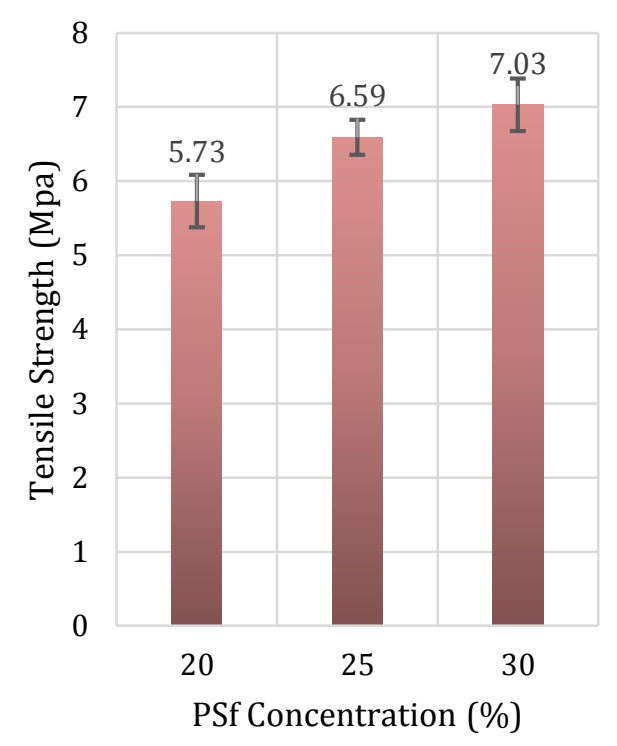

Figure 3 Tensile Strength of Asymmetric PSf Membrane at Different Polymer Concentration

Basically, the increased in the tensile strength of the membrane is due to the improvement in the morphological structure of the membrane. As the polymer concentration increased, the formation of macro-voids structure become lesser and smaller which improve the structure of the membrane as observed in Figure 2 [17-18]. As a result, the tensile strength of the membrane also increases. Moreover, the increase in polymer concentration affected the tensile strength due to the fewer formation of macro-voids [19].

The change in elongation at break different polymer concentration is presented in Figure 4. The results for different PSf concentration shows a slightly different trend compared to the tensile strength. Initially, the elongation at break increases when the polymer concentration is increased from $20 \mathrm{wt} \%$ to $25 \mathrm{wt} \%$ similar to the tensile strength. Then, at higher polymer concentration of $30 \mathrm{wt} \%$, the elongation at break decreases, 
contradicting to the tensile strength result. Since elongation at break represent the how long the membrane can be stretched before it breaks, this phenomena happened likely due to the rigidified membrane structure with increasing polymer concentration. Thus, the decreased in elongation is observed.

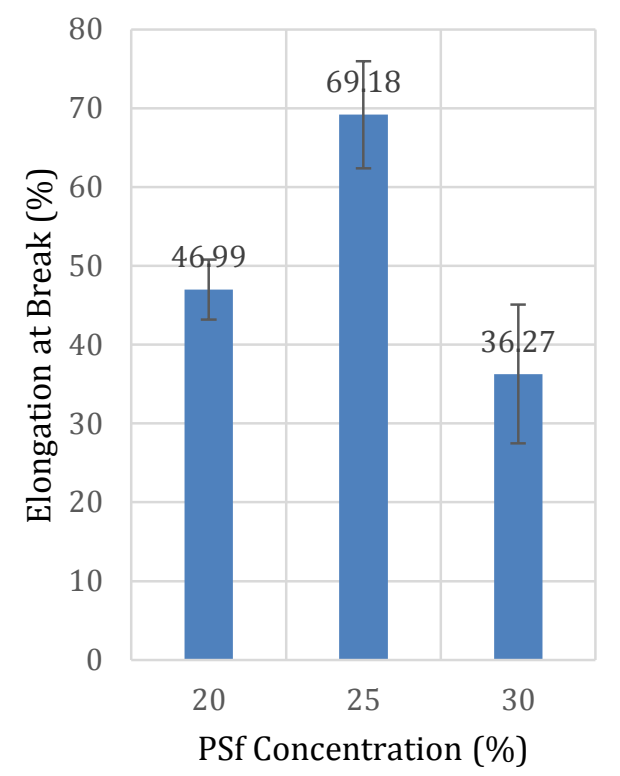

Figure 4 Elongation at Break of Asymmetric PSf Membrane at Different Polymer Concentration

\subsection{CONCLUSION}

In this study, asymmetric PSf/NMP membranes were successfully fabricated with three different polymer concentrations ranging from $20 \mathrm{wt} \%$, to $30 \mathrm{wt} \%$. The viscosity analysis showed that as the polymer concentration is increased, the dope solution viscosity is also increased. Moreover, from the extrapolation of the dope viscosity, the optimum polymer concentration obtained was 26 wt $\%$ PSf. Furthermore, the SEM micrographs showed that an addition in polymer concentration from 20 to 30 wt $\%$ changed the morphological structure of the membrane, from a thin skin layer with large finger-like macrovoids structure to a denser skin layer with rounder tear-drop macrovoids structure. In addition, this study experimental result also revealed that there is an improvement in the mechanical properties. The tensile strength of the membrane increases as the polymer concentration is increased. Finally, this study concluded that the increasing polymer concentration significantly improves the morphological and mechanical properties of the membrane.

\section{ACKNOWLEDGEMENT}

The authors would like to extend their gratitude to Universiti Malaysia Sabah (UMS) for the research fund through Research University Grant vot no. SBK0290-TK-2016. Also, we sincerely thanked Faculty of Engineering UMS as well as Advanced Membrane Technology Research Centre (AMTEC) UTM for their assistance towards completion of this project.

\section{REFERENCES}

[1] B. Shimekit and H. Mukhtar. 2012. Natural Gas Purification Technologies-Major $\quad \mathrm{CO}_{2}$ Separation and Future Directions. Universiti of Teknologi Petronas. 235-270.

[2] S. Mokhatab and W. Poe. 2012. Handbook of Natural Gas Transmission and Processing. Second Edition. Elsevier B.V. 253-290.

[3] D. F. Sanders, Z. P. Smith, R. Guo, L. M. Robeson, J. E. McGrath, D. R. Paul and B. D. Freeman. 2013. Energy Efficient Polymeric Gas Separation Membranes for a Sustainable 
Future: A Review. J. Polym. 54: 4729-4761.

[4] R. Abedini and A. Nezhadmoghadam. 2010. Application of Membrane in Gas Separation Processes: Its Suitability and Mechanisms. $J$. Pet. Coal. 52: 69-80.

[5] N. M. Ismail, A. F. Ismail, A. Mustafa, T. Matsuura, T. Soga, K. Nagata, T. Asaka. 2015. Qualitative and Quantitative Analysis of Intercalated and Exfoliated Silicate Layers in Asymmetric

Polyethersulfone/Cloisite15a Ò Mixed Matrix Membrane for $\mathrm{CO}_{2} / \mathrm{CH}_{4}$ Separation. Chem. Eng. J. 268: 371-383.

[6] S. Hamzah, N. Ali, M. M. Ariffin, A. Ali and A. W. Mohammad. 2014. High Performance of Polysulfone Ultrafiltration Membrane: Effect of Polymer Concentration. $J$. Eng. Appl. Sci. 9: 2543-2550.

[7] H. Julian and I. G. Wenten. 2012. Polysulfone Membranes for $\mathrm{CO}_{2} / \mathrm{CH}_{4}$ Separation: State of the Art. J. Eng. 2: 484-495.

[8] A. K. Hołda, B. Aernouts, W. Saeys and I. F. J. Vankelecom. 2013. Study of Polymer Concentration and Evaporation Time as Phase Inversion Parameters for Polysulfonebased SRNF Membranes. $J$. Memb. Sci. 442: 196-205.

[9] A. F. Ismail and L. P. Yean. 2003. Review on the Development of Defect Free and Ultrathin Skinned Asymmetric Membranes for Gas Separation Through Manipulation of Phase Inversion and Rheological Factors. J. Appl. Polym. Sci. 88: 442-451.

[10] M. T. Ravanchia, T. Kaghazchia and A. Kargarib. 2009. Application of Membrane
Separation Processes in Petrochemical Industry: A Review. Desalination. 235: 199244.

[11] N. M. Ismail, A. F. Ismail and A. Mustafa. 2015. Sustainability in Petrochemical Industry: Mixed Matrix Membranes from Polyethersulfone/cloisite $15 \mathrm{~A}^{\circledR}$ for the Removal of Carbon Dioxide. Procedia CIRP. 26: 461-466.

[12] N. Peng, T. S. Chung and K. Y. Wang. 2014. Macrovoid Evolution and Critical Factors to Form Macrovoid-free Hollow Fiber Membranes. J. Memb. Sci. 318: 363-372.

[13] T. S. Chung, S. K. Teoh, and X. Hu. 1997. Formation of Ultrathin Highperformancepolyethersulfone Hollow-fiber Membranes. $J$. Memb. Sci. 133: 161-175.

[14] M. I. Mustaffar, A. F. Ismail and R. M. Illias. 2004. Study on the Effect of Polymer Concentration on Hollow Fiber Ultrafiltration Membrane Performance and Morphology. Reg. Conf. Eng. Educ. RCEE 2005. 1-12.

[15] B. Zhou and Ph.D. Massachusetts Institute of Technology. 2006. Simulations of Polymeric Membrane Formation in 2D and 3D.

[16] H. Sofiah, A. Nora'aini and M. A. Marinah. 2010. The Influence of Polymer Concentration on Performance and Morphology of Asymmetric Ultrafiltration Membrane for Lysozyme Separation. J. Appl. Sci. 10: 3325-3330.

[17] M. Z. Yunos, Z. Harun, H. Basri, A. F. Ismail. 2012. Effects of Water as Non-solvent Additive on Performance of Polysulfone Ultrafiltration Membrane. $A d v$. Mater. Res. 488-489: 46-50. 
[18] X. Wang, Z. Li, M. Zhang, T. Fan and B. Cheng. 2017. Preparation of a Polyphenylene Sulfide Membrane from a Ternary Polymer/Solvent/NonSolvent System by Thermally Induced Phase Separation. $R$.
Soc. Chem. 7: 10503-10516.

[19] P. Amirabedi, R. Yegani and M. K. R. Aghjeh. 2013. Experimental Design Applied toFabrication of PSf Membranes via NIPS Method. J. Text. Polym. 1: 24-30. 